\title{
Development of uniform CdTe pixel detectors based on Caltech ASIC
}

Kousuke Oonuki, Hokuto Inoue, Kazuhiro Nakazawa, Takefumi Mitani, Takaaki Tanaka, et al.

Kousuke Oonuki, Hokuto Inoue, Kazuhiro Nakazawa, Takefumi Mitani, Takaaki Tanaka, Tadayuki Takahashi, C. M. Hubert Chen, Walter R. Cook, Fiona A. Harrison, "Development of uniform CdTe pixel detectors based on Caltech ASIC," Proc. SPIE 5501, High-Energy Detectors in Astronomy, (29 September 2004); doi: 10.1117/12.552598

Event: SPIE Astronomical Telescopes + Instrumentation, 2004, Glasgow, United Kingdom 


\title{
Development of Uniform CdTe Pixel Detectors Based on Caltech ASIC
}

\author{
Kousuke Oonuki ${ }^{a, b}$, Hokuto Inoue $^{a, b}$, Kazuhiro Nakazawa $^{a}$, Takefumi Mitani ${ }^{a, b}$, \\ Takaaki Tanaka ${ }^{a, b}$, Tadayuki Takahashi ${ }^{a, b}$, C. M. Hubert Chen $^{c}$, \\ Walter R. Cook $^{c}$, and Fiona A. Harrison ${ }^{c}$ \\ ${ }^{a}$ Institute of Space and Astronautical Science (ISAS/JAXA), Sagamihara, Kanagawa \\ 229-8510, Japan \\ ${ }^{b}$ Department of Physics, University of Tokyo, Bunkyo, Tokyo 113-0033, Japan \\ ${ }^{c}$ Department of Physics, California Institute of Technology, Pasadena, CA 91125, USA
}

\begin{abstract}
We have developed a large CdTe pixel detector with dimensions of $23.7 \times 13.0 \mathrm{~mm}^{2}$ and a pixel size of $448 \times 448$ $\mu \mathrm{m}^{2}$. The detector is based on recent technologies of an uniform CdTe single crystal, a two-dimensional ASIC, and stud bump-bonding to connect pixel electrodes on the CdTe surface to the ASIC. Good spectra are obtained from 1051 pixels out of total 1056 pixels. When we operate the detector at $-50{ }^{\circ} \mathrm{C}$, the energy resolution is $0.67 \mathrm{keV}$ and $0.99 \mathrm{keV}$ at $14 \mathrm{keV}$ and $60 \mathrm{keV}$, respectively. Week-long stability of the detector is confirmed at operating temperatures of both $-50^{\circ} \mathrm{C}$ and $-20{ }^{\circ} \mathrm{C}$. The detector also shows high uniformity: the peak positions for all pixels agree to within $0.82 \%$, and the average of the energy resolution is $1.04 \mathrm{keV}$ at a temperature of $-50{ }^{\circ} \mathrm{C}$. When we normalized the peak area by the total counts detected by each pixel, a variation of $2.1 \%$ is obtained.
\end{abstract}

Keywords: CdTe, CZT, uniformity, hard X-ray detector, pixel detector, stud bump bonding, imaging spectrometer

\section{INTRODUCTION}

One of the primary fields of high energy astro-physics in the near future is the hard X-ray universe, where nonthermal processes such as particle acceleration and nucleo-systhesis become dominant. The combination of the two new technologies of hard X-ray focusing mirror optics ${ }^{1}$ and hard X-ray imaging spectrometers ${ }^{2}$ at the focal plane will provide two orders of magnitude improvement in both detection sensitivity and imaging resolution..$^{3-5}$ For example, the NeXT mission ${ }^{3,6}$ proposed in Japan has mirrors with a focal length of $12 \mathrm{~m}$ and an angular resolution up to 15 arcsec. $^{7}$ The detector is required to have an energy coverage from $5 \mathrm{keV}$ to $80 \mathrm{keV}$, with an energy resolution better than $1.0 \mathrm{keV}$ (FWHM) for $60 \mathrm{keV}$ line $\gamma$-rays. ${ }^{8}$ An aperture size of $20-30 \mathrm{~mm}$ in diameter with a spacial resolution of $200-250 \mu \mathrm{m}$ is needed to take advantage of the performance of the mirror. The detector should have a good timing resolution in the range $10-100 \mu \mathrm{s}$ on an event by event basis to reduce the intrinsic background in the detector by the anti-coincidence technique. Good uniformity in both the detection efficiency and the spectroscopic properties is necessary not only to obtain high quality image and spectra, but also to perform proper background subtraction.

Cadmium telluride (CdTe) and cadmium zinc telluride (CZT) are promising devices as the focal plane detector since they have a high detection efficiency comparable to NaI scintilators, a good energy resolution comparable to Ge detectors, and can be operated at room temperature. Thanks to significant progress on technologies of crystal growth, large area detectors based on CdTe and CZT are now available. Recently, Harrison et al. has developed a large area CZT pixel detector with a newly developed low-noise ASIC for the front end. ${ }^{9}$ The detector shows a good energy resolution of $<1 \mathrm{keV}$. However, the present high pressure Bridgeman method, which is often used to grow CZT crystal, only yields polycrystals and therefore the yield of obtaining large $(>1$

Further author information: (Send correspondence to K.O.)

K.O.: E-mail: oonuki@astro.isas.jaxa.jp, Telephone: 81427598510 
$\mathrm{cm}^{2}$ ) and uniform portions of single CZT crystals is low. To control the mobility and carrier lifetime within the whole wafer seems to be difficult. ${ }^{10,11}$ This non-uniformity is the issue of the current CZT pixel detectors. On the other hand, CdTe crystal grown by the Traveling Heater Method (THM-CdTe) can provide a single crystal as large as $40 \mathrm{~mm} \times 40 \mathrm{~mm} .^{12}$ Based on the THM-CdTe wafers, we have been working on high performance CdTe detectors for both planar and pixel configuration. ${ }^{13-16}$

In order to improve the current hard X-ray imaging detector by utilizing the recent CdTe technology, we have developed a pixel detector under the collaboration of ISAS and Caltech. In this collaboration, a two-dimensional large area analog ASIC and the read out system is prepared by Caltech. A large size CdTe crystal with pixel electrodes and the bump bonding technologies are prepared by ISAS. In this paper, uniformity of the pixel detector as well as basic performance of the CdTe pixel detector is presented.

\section{CDTE PIXEL DETECTOR}

The uniform charge transport properties of the wafer are very important for fabricating large area pixel detectors. The CdTe crystal used here is the THM-CdTe manufactured by ACRORAD. ${ }^{12}$ An electrical resistivity of $\sim 1 \times 10^{9}$ $\Omega \mathrm{cm}$ (p-type) is achieved by compensating the native defects with $\mathrm{Cl}$. The grown crystal is large enough to obtain ( 1111$)$ - oriented single crystal wafers with an area as large as $25 \times 25 \mathrm{~mm}^{2}$. We have demonstrated that the wafer shows very good uniformity when we make a large area CdTe detector with planar electrodes. ${ }^{13,14}$ As determined by the measurements with a large planar detector $\left(21 \times 21 \mathrm{~mm}^{2}\right)$ by using a collimated $\gamma$-ray beam from an ${ }^{241} \mathrm{Am}$ source, the location of the peak in the pulse height distribution agrees to within $0.1 \%$ and the variation of the area of the $60 \mathrm{keV}$ peak is less than $0.9 \%$, regardless of the position.

A schematic drawing of the detector is shown in Fig. 2. The detector consists of a CdTe wafer, a frontend ASIC and the data handling circuit including an ADC and a CPU. Charge signals from the individual pixel electrode are fed into the readout circuit built in the ASIC. The ASIC is originally developed by the Caltech group for a CZT pixel detector and features very low noise by means of a capacitor array. ${ }^{17}$ The power consumption of the ASIC is as low as $50 \mu \mathrm{W} /$ pixel.

Before assembling the detector, a CdTe wafer with dimensions of $23.7 \times 13.0 \mathrm{~mm}^{2}$ and a thickness of $0.5 \mathrm{~mm}$ is prepared. By using polished wafers with (l 111 ) orientation, Pt electrodes are formed by electroless plating for both the common electrode (cathode) and pixel electrodes (anode). The size of each pixel electrode is 448 $\mu \mathrm{m} \times 448 \mu \mathrm{m}$ with a gap of $52 \mu \mathrm{m}$. No control electrode is adopted between electrodes. A guard with $1 \mathrm{~mm}$ width surrounds the $24 \times 44$ pixels anode pixels.

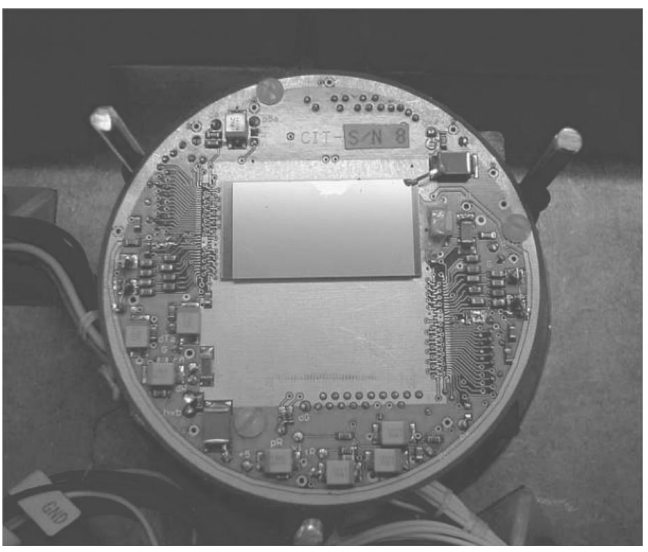

Figure 1. Photo of the pixel detector. The detector has dimensions of $23.7 \times 13.0 \mathrm{~mm}^{2}$ and a thickness of $0.5 \mathrm{~mm}$. The cathode surface is shown in the picture. The bias voltage is supplied from the top board to the cathode.

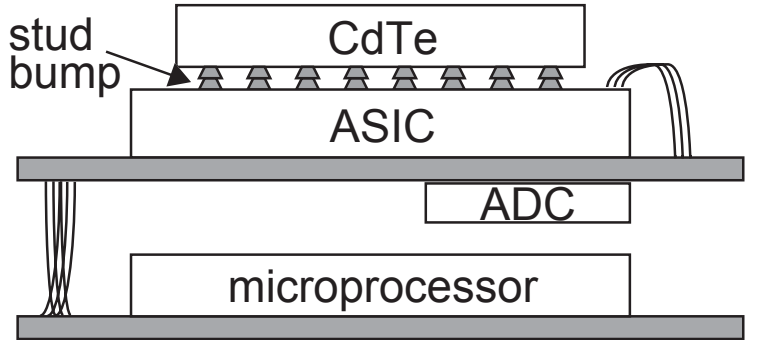

Figure 2. Schematic drawing. The detector consists of CdTe, ASIC, stud bumps between them, an off-chip $\mathrm{ADC}$, and a microprocessor for the readout logic. 
(a)

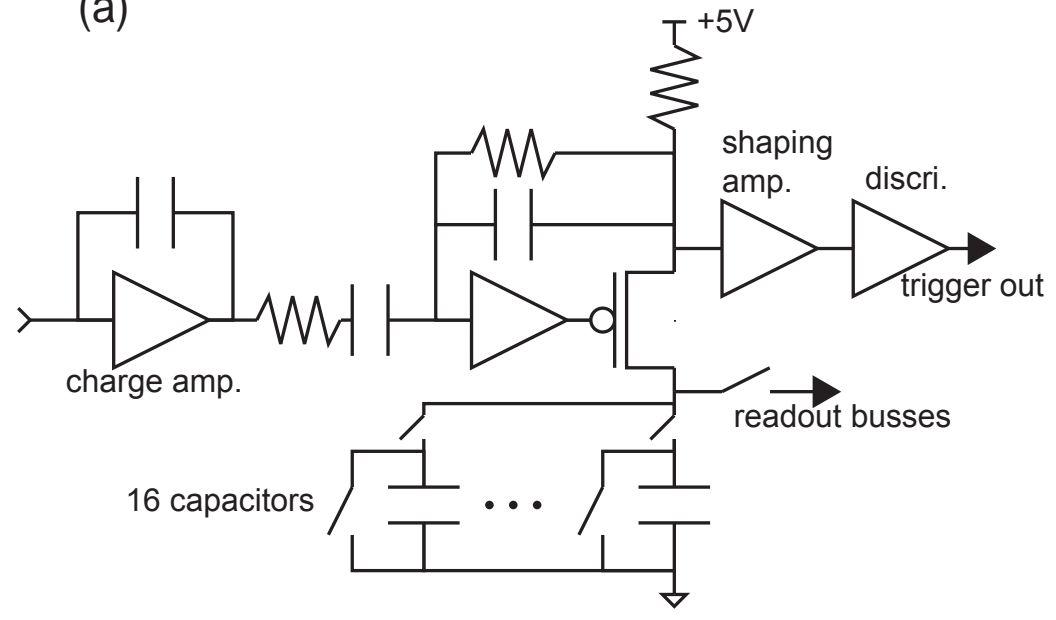

(b)

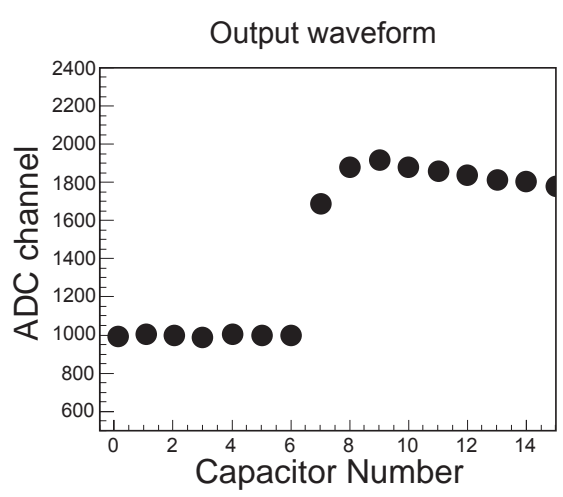

Figure 3. (a) Schematic diagram of the the circuit for one pixel which is implemented in the ASIC, and (b) An example of an output waveform. The circuit has a charge amplifier, a shaping amplifier, a discriminator, and a capacitor array of 16 capacitors. The outputs are sixteen samplings by the array of capacitors.

One of the most difficult parts to realize a fine pitch (finer than several hundred microns) CdTe and/or CZT pixel detectors is to establish a simple and robust connection technology for these fragile devices. High compression and/or high ambient temperature would damage the crystal. Also the co-planarity of the CdTe wafer is measured to be $2 \mu \mathrm{m}$ at most, which is much worse than that of usual silicon wafers. Usual indium-ball soldering might not be appropriate for this purpose. Also indium is easily oxidizable, and needs flux which could contaminate the detector surface. Recently, we have developed indium and gold (In/Au) stud-bump bonding technology, optimized for CdTe detectors, with Mitsubishi Heavy Industries (MHI) in Japan. By following the prescription given in Takahashi et al., ${ }^{18}$ but with a slightly modified bump condition, we connected the CdTe wafer to the ASIC. In order to minimize the effect of incomplete charge collection, we apply a negative bias on the common electrode. After we assembled the detector, we have confirmed the connection of 1055 pixels out of 1056 pixels. There is one bad pixel which shows very high noise, regardless of any conditions, such as bias voltage, and temperature. Currently, we do not understand whether the read out circuit is broken, or the bump is not connected for this particular pixel.

In the ASIC (Fig. 3 (a)), output signal from the preamplifier is continuously integrated by one capacitor to the next with a time interval of $1 \mu \mathrm{s}$. After a trigger signal is issued from a comparator, the switching sequence continues for the next eight capacitors and then the sequence is freezed for the subsequent readout. The charge in sixteen capacitors is sequentially read out as voltage and converted by the external ADC. Figure 3 (b) shows an example of the output signal for one pixel. Pulse height information for each X-ray photon is calculated by analyzing the recorded pulse shape by the filter algorithm implemented in DSP or by software. The ASIC also reads out data from the surrounding eight pixels together with the data from the triggered pixel. A detailed description of the ASIC is given in Chen et al. ${ }^{17}$ For the detector used in this experiment, triggers from five pixels are turned off, because these channels show rather high noise as compared to other channels. The typical noise level is about 50 ADC channel for normal pixels, while the noise level of bad channels is larger than 200 ADC channel. From hereafter, we analyze spectra from 1051 pixels out of total 1056 pixels. At $-20{ }^{\circ} \mathrm{C}$, the FWHM of the peak measured by the test pulse ranges from $0.4 \mathrm{keV}$ to $0.6 \mathrm{keV}$ when the bias voltage is not applied. This corresponds to the electronic noise of $40-60 e^{-}$(RMS).

The spectrum from each pixel is constructed from the pulse height information associated with the trigger. There are two types of events; 1) single-pixel events in which the pulse height of pixels surrounding the triggered pixel is comparable with the noise level, and 2) multi-pixel events in which the energy deposited in the CdTe detector is shared with multiple pixels. 


\section{SIGNAL PROCESSING AND SPECTRAL ANALYSIS}

In order to study the spectral performance of the CdTe pixel detector, we start from the search for the optimum operating condition and the investigation of the response from a single pixel. The overall performance, including the pixel by pixel variation will be discussed in the next section.

As the first demonstration of the operation of the CdTe pixel detector, Fig. 4 shows ${ }^{241}$ Am spectra taken at $-20^{\circ} \mathrm{C}$ and $-50{ }^{\circ} \mathrm{C}$. Single-pixel events are used to make the spectra. Energy resolutions better than $1 \mathrm{keV}$ (FWHM) are obtained for $\gamma$-ray lines from $14 \mathrm{keV}$ to $60 \mathrm{keV}$ in the spectra. The best resolution is obtained when the detector is operated at the lowest temperature, $-50{ }^{\circ} \mathrm{C}$. With a bias voltage of $100 \mathrm{~V}$, the energy resolution at $14 \mathrm{keV}$ and $60 \mathrm{keV}$ is $0.67 \mathrm{keV}$ and $0.89 \mathrm{keV}$, respectively. The improvement in the spectral resolution is due to the reduction of the leakage current. At $-20{ }^{\circ} \mathrm{C}$, the leakage current is estimated to be an order of $10 \mathrm{pA}$ per pixel at $-100 \mathrm{~V}$, which is calculated from the results obtained with a planar detector with $\mathrm{Pt} / \mathrm{CdTe} / \mathrm{Pt}$ electrode configuration. Although we need to perform more quantitative measurements, the leakage current at $-50{ }^{\circ} \mathrm{C}$ is expected to be reduced by almost an order of magnitude as compared to that at $-20{ }^{\circ} \mathrm{C}$.

Charge collection efficiency (CCE) is an important issue for CdTe and CZT, because electrons and holes generated in these semiconductor have low mobility and a short life-time. ${ }^{19}$ The low CCE results in the tail-like structure below the peak in the spectrum, which is actually seen in the ${ }^{241} \mathrm{Am}$ spectra (Fig. 4). We study the CCE quantitatively by measuring the ${ }^{57} \mathrm{Co}$ spectrum at different bias voltages under an operating temperature of $-50{ }^{\circ} \mathrm{C}$. As clearly shown in the case of the $40 \mathrm{~V}$ operation (Fig. 5), a significant amount of charge loss is seen as a low-energy tail of the $122 \mathrm{keV}$ line. As the bias voltage is increased to $300 \mathrm{~V}$, a sharp $122 \mathrm{keV}$ peak is obtained. A higher bias voltage is better in terms of the CCE because the fraction of electrons and holes that reach the electrodes increases. However, high bias voltage deteriorates the spectral resolution due to the increase of the leakage current. In the measurements, the best energy resolution for the $14 \mathrm{keV}$ line is obtained at $40 \mathrm{~V}$. On the other hand, the best energy resolution of $1.47 \mathrm{keV}$ for the $122 \mathrm{keV}$ line is obtained at $300 \mathrm{~V}$. This is because most of the $14 \mathrm{keV}$ photons interact close to the cathode electrodes, and because the transit of electrons, which is less affected by charge loss, becomes responsible for the pulse height. As an optimum value, we use $300 \mathrm{~V}$ as the standard bias voltage from here after.

Since the pixel size is as small as $500 \mu \mathrm{m}$ for the CdTe pixel detector, we need to investigate the charge splitting among the adjacent pixels (multi-pixel event). Figure 6 shows a correlation of pulse heights between a triggering pixel and one of the adjacent pixels. It is shown that the energy deposition, corresponds to the $122 \mathrm{keV} \gamma$-ray, is shared between two pixels, for some fraction. Figure 7 compares spectra for single-pixel and multi-pixel events. It is clearly shown that the amount of the tail component is more pronounced when we include multi-pixel events. This implies that the selection of single-pixel events is important to obtain "tail-less" spectra in the CdTe pixel detector.
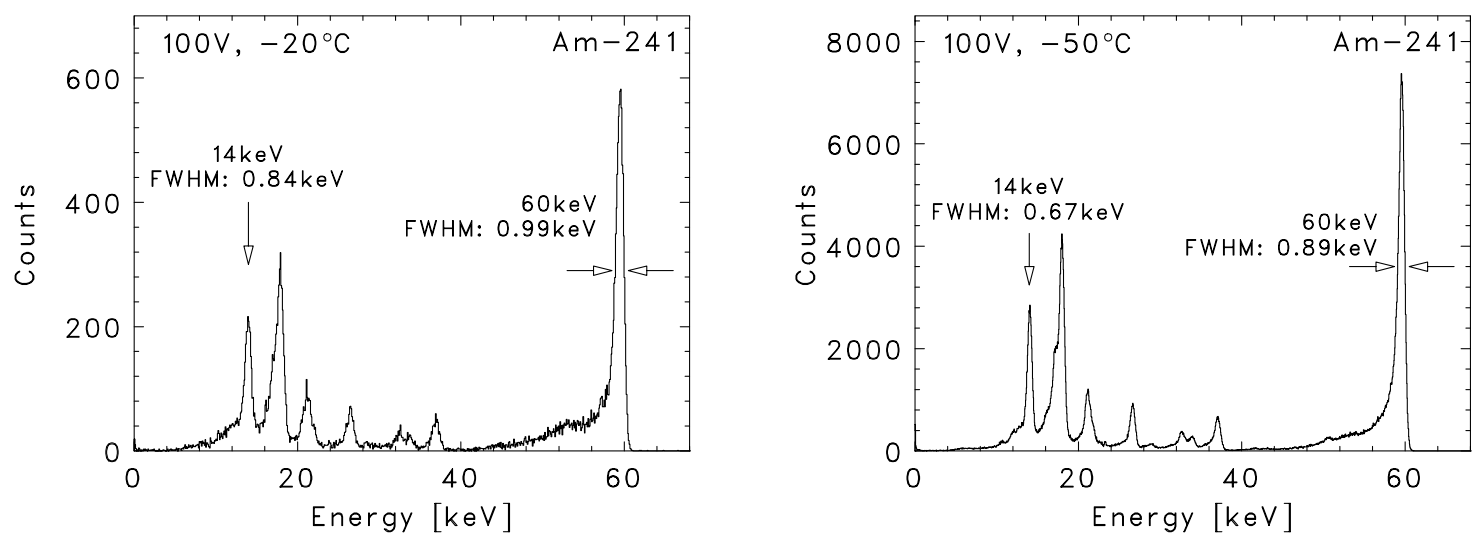

Figure 4. ${ }^{241} \mathrm{Am}$ spectra obtained at $-20{ }^{\circ} \mathrm{C}$ and $-50{ }^{\circ} \mathrm{C} .{ }^{241} \mathrm{Am}$ illuminates the detector from $40 \mathrm{~cm}$ above the cathode plane of the detector. 


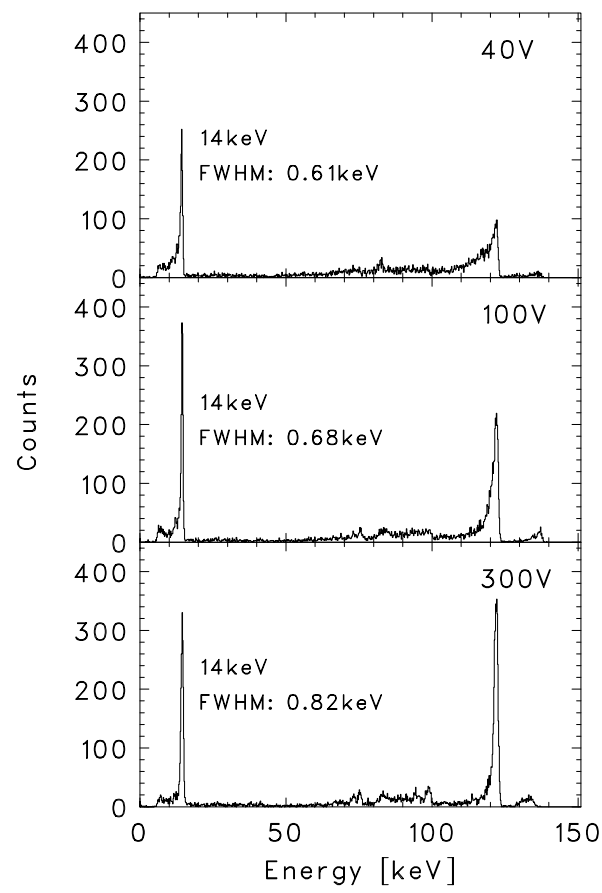

Figure 5. ${ }^{57} \mathrm{Co}$ spectrum obtained at an operating temperature of $-50{ }^{\circ} \mathrm{C}$ with different bias voltages. The applied bias is $40 \mathrm{~V}, 100 \mathrm{~V}$, and $300 \mathrm{~V}$. All the spectra are drawn by using singlepixel events in which no signal larger than $5 \mathrm{keV}$ is detected in the surrounding pixels. The energy resolution for the $14 \mathrm{keV}$ line is as low as $0.61 \mathrm{keV}$ at the bias voltage of $40 \mathrm{~V}$. The size of the low-energy tail is reduced as the bias voltage increases.

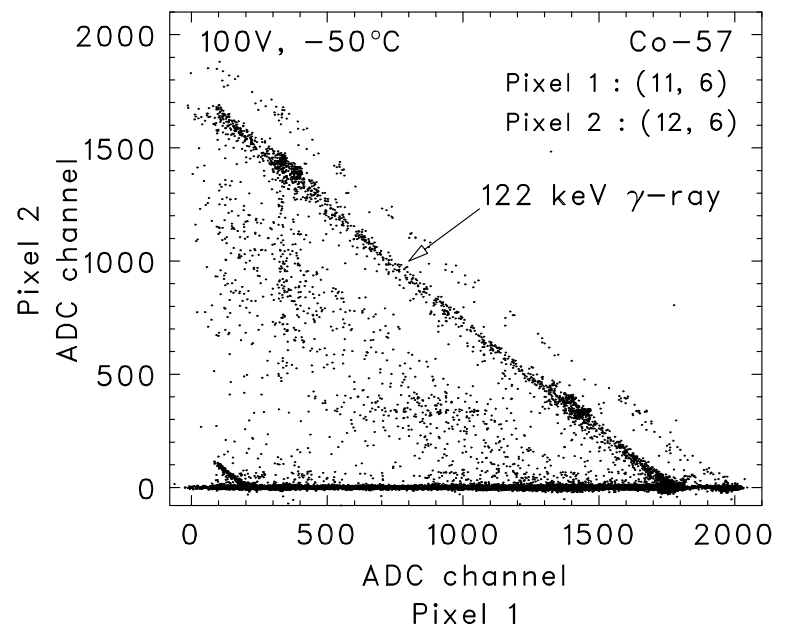

Figure 6. Correlation between pulse height (ADC channel) from a triggering pixel and that of the adjacent pixel. The horizontal axis is the ADC channel for the triggered pixel while the vertical axis for the adjacent pixel.

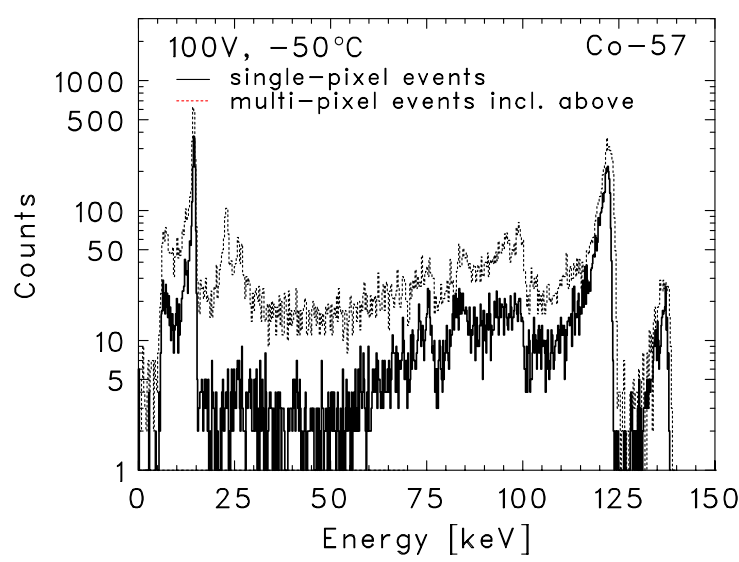

Figure 7. Spectra both for single-pixel events (black) and multi-pixel events including single-pixel events (dash). A low energy threshold of $5 \mathrm{keV}$ is used to discriminate hits in the surrounding pixels. 
Long-term stability is an important issue for CdTe and CZT semiconductors. ${ }^{18}$ As discussed in our previous publication, ${ }^{13}$ a CdTe detector with Pt electrode for both cathode and anode sides (Pt/CdTe/Pt) shows good stability. We do not see any spectral degradation for a long term operation of a week or more. In order to verify this with the CdTe pixel detector, we operate it for seven days at an operating temperature of $-50{ }^{\circ} \mathrm{C}$ under a bias of $300 \mathrm{~V}$. An ${ }^{241} \mathrm{Am}$ source is placed above the detector during the measurement. Figure 8 shows the spectra taken at the first day, 1 day later, and 7 days later. No degradation can be observed in the peak positions and the energy resolution. The location of the peak in the pulse height distribution agrees to within $0.2 \%$. The energy resolutions (FWHM) agree within statistical errors. The detector also shows a stable spectral performance at $-20{ }^{\circ} \mathrm{C}$ over one week.

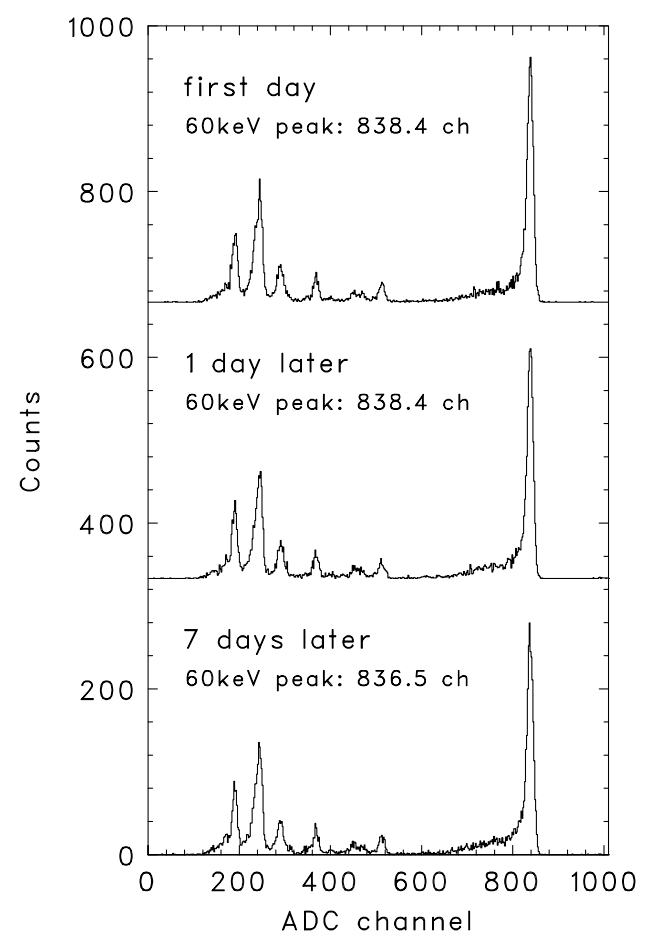

Figure 8. Spectra taken at the first day, 1 day later, and a week later after the bias voltage is applied. An operating temperature is $-50{ }^{\circ} \mathrm{C}$ and a bias voltage is $300 \mathrm{~V}$. The spectra are drawn by summing 10 pixels.

\section{UNIFORMITY OF THE DETECTOR}

Evaluating the detector uniformity response is an important objective of this experiment. We analyze the uniformity of the detector in terms of spectral properties such as the peak position and the spectral resolution, as well as the detection efficiency.

The location of the peak in the spectrum for mono-energetic $\gamma$-rays has a strong correlation with the charge transport properties of the material, ${ }^{10,19}$ especially for electrons. If the mobility-lifetime product is not uniform in the wafer used in the detector, the peak position changes with the pixel location, since we apply a single bias voltage over the entire detector plane. The variations of the size of low-energy tail is explained if the transport properties for holes have some spatial distribution. These spatial variations of charge transport properties could be introduced if the crystal is not uniformly grown.

We study the uniformity by irradiating with an ${ }^{241} \mathrm{Am}$ source from $40 \mathrm{~cm}$ above the detector, and obtain flat illumination data. The detector is operated under a bias of $300 \mathrm{~V}$, at a temperature of $-50{ }^{\circ} \mathrm{C}$. In order to evaluate the charge transport properties, the analog gains for each pixel are calibrated by the using the on-chip 


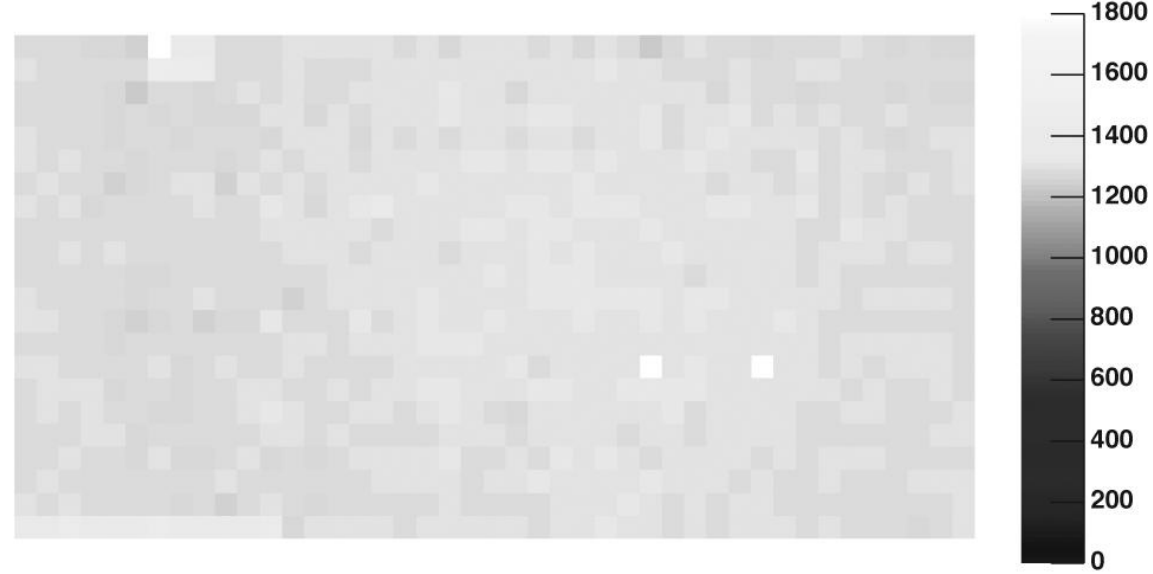

Figure 9. Map of the peak positions of the $60 \mathrm{keV}$ line. The white spots at the right center and top left are disabled pixels.

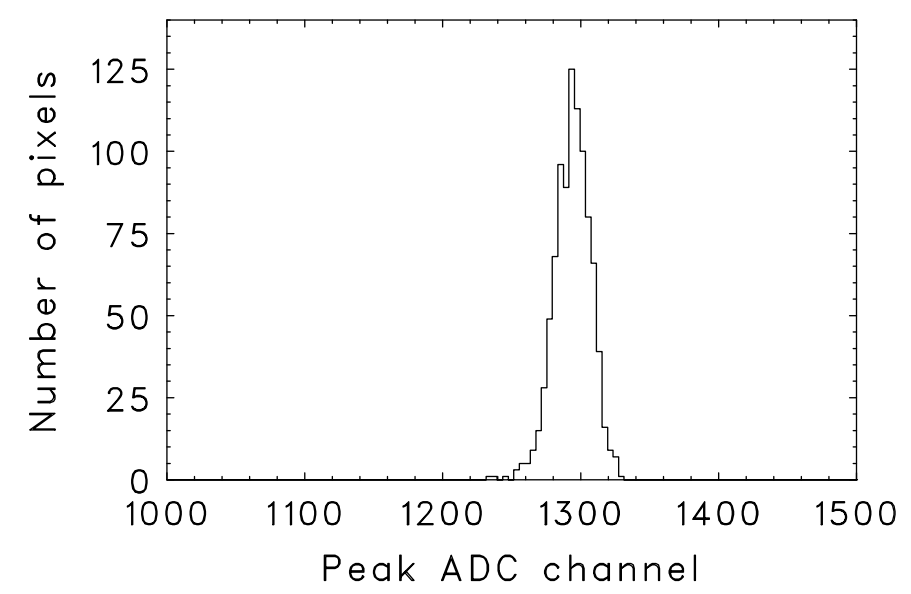

Figure 10. Distribution of the $60 \mathrm{keV}$ peak positions after the analog gain of the ASIC is calibrated by means of pulser data. The variation for the peak position is less than $0.82 \%$.

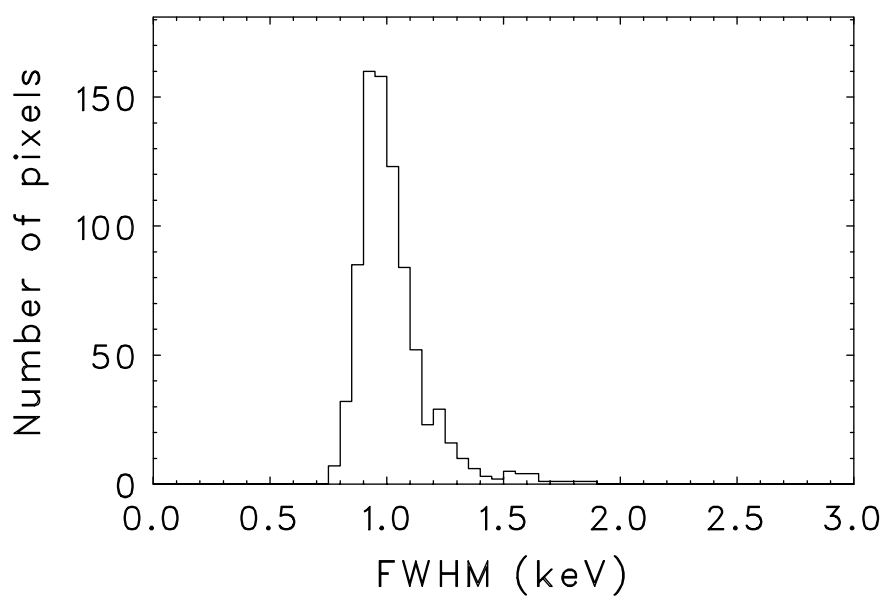

Figure 11. Distribution of the energy resolution (FWHM) for the $60 \mathrm{keV}$ line. The mean and standard deviation of the distribution are $1.04 \mathrm{keV}$, and $0.10 \mathrm{keV}$. 
test pulse. The average gain variation is $2.8 \%$, consistent with the number reported in Harrison et al. ${ }^{20}$ Figure 9 shows the spatial distribution of the peak position in ADC channel after the gain of readout circuit in the ASIC is calibrated. Distribution of the peak position is almost flat and shows a standard deviation of $0.82 \%$ (Fig. 10), which agrees with the result from the scanning experiment of a planar CdTe detector. ${ }^{14,18}$ Distribution of the energy resolution is also presented in Fig. 11. The average energy resolution is $1.04 \mathrm{keV}$, and the standard deviation is $0.10 \mathrm{keV}$.

The variation of the count rate is also an important aspect for the pixel detector, since it reflects the variations of the detection efficiency in the detector. In order to produce precise-spectroscopic images, the flat efficiency in the detector plane is desirable to reduce the systematic errors. The uniformity of charge transport properties is again important here. This is because the pixel-to-pixel variation of the fraction of low energy tail affects the flatness of the image when we specify the energy band. Distribution of the area for the $60 \mathrm{keV}$ peak is plotted in Fig. 12. The area is calculated by integrating counts from $45 \mathrm{keV}$ to $65 \mathrm{keV}$. The variation of the distribution is $8.9 \%$, which is larger than the statistical variation of $1.1 \%$. This result is inconsistent with the result for the planar CdTe detector. ${ }^{14,18}$

In order to investigate the reason for the variation of the area, we compare the counts in the $60 \mathrm{keV}$ peak and the total count, and obtain a scatter plot (Fig. 13). From the figure, a strong correlation between the two values is observed. In Fig. 14, we compared the actual spectra of the pixels with the highest and the lowest count at 60

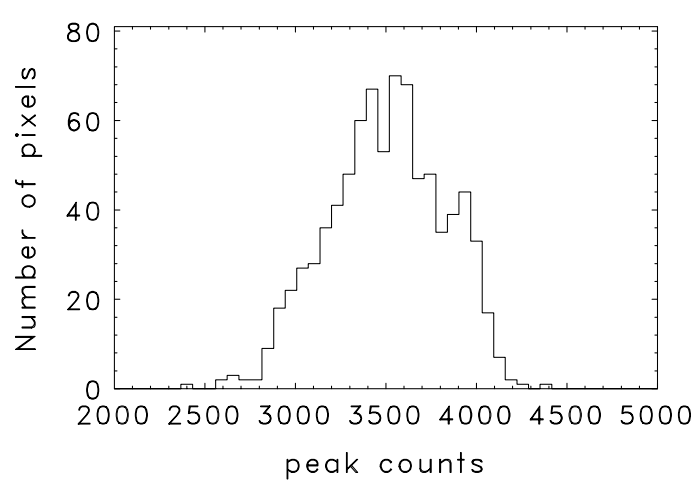

Figure 12. Count variation for the $60 \mathrm{keV}$ peak. The variation is $8.9 \%$.

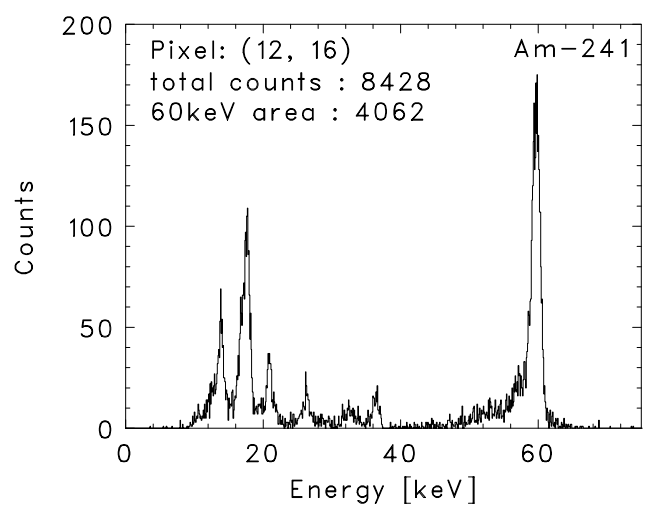

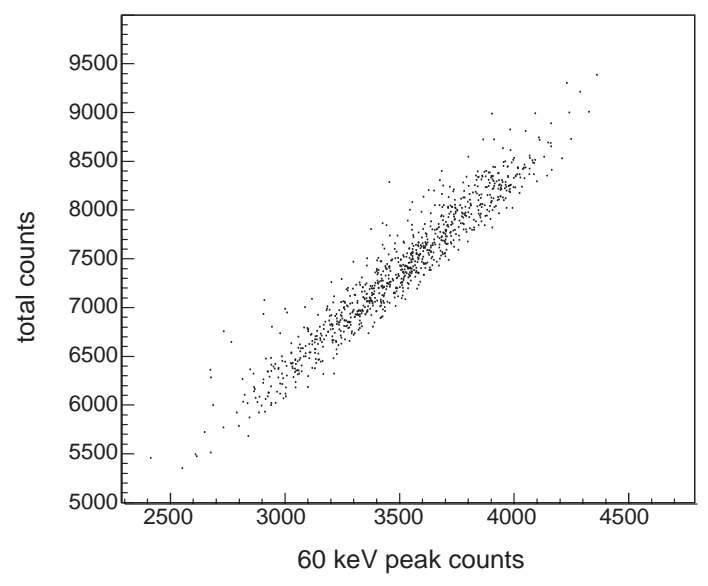

Figure 13. Correlation between $60 \mathrm{keV}$ peak counts and total counts for each pixel.

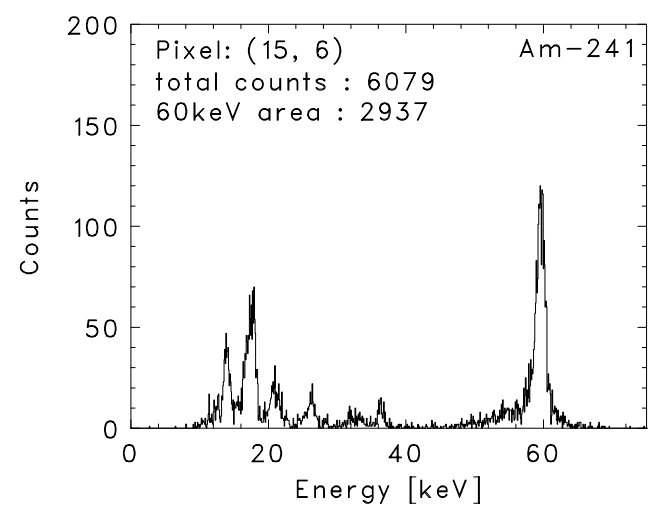

Figure 14. Spectra for the two extremes of the total counts. 


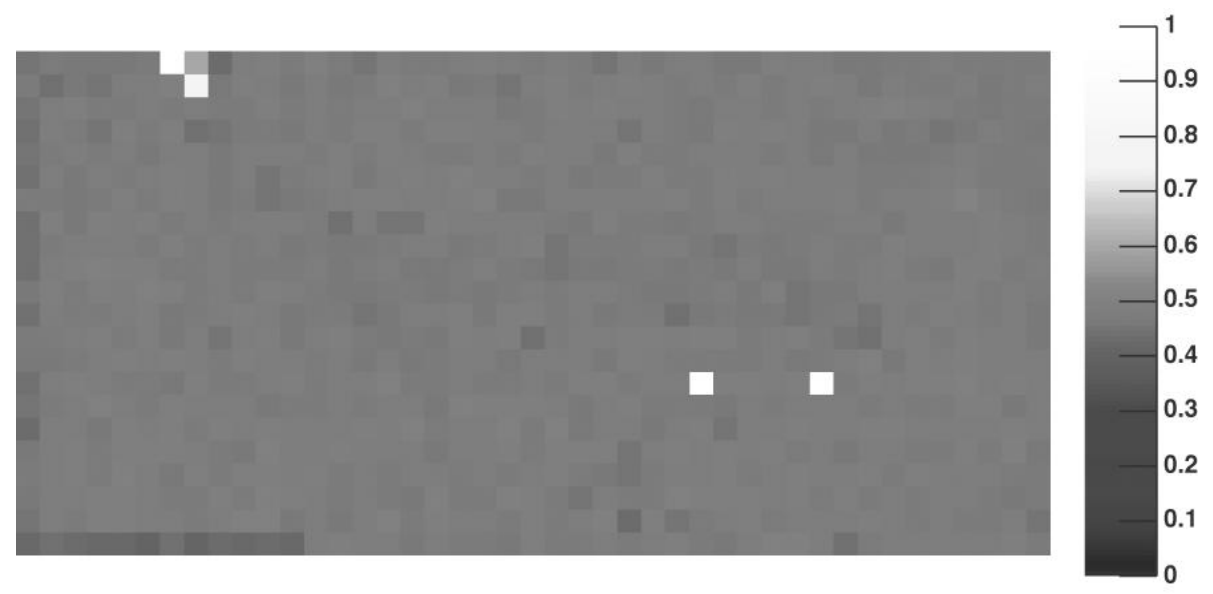

Figure 15. Map for the $60 \mathrm{keV}$ peak area normalized by the total counts detected by each pixel. The white spots are disabled pixels.

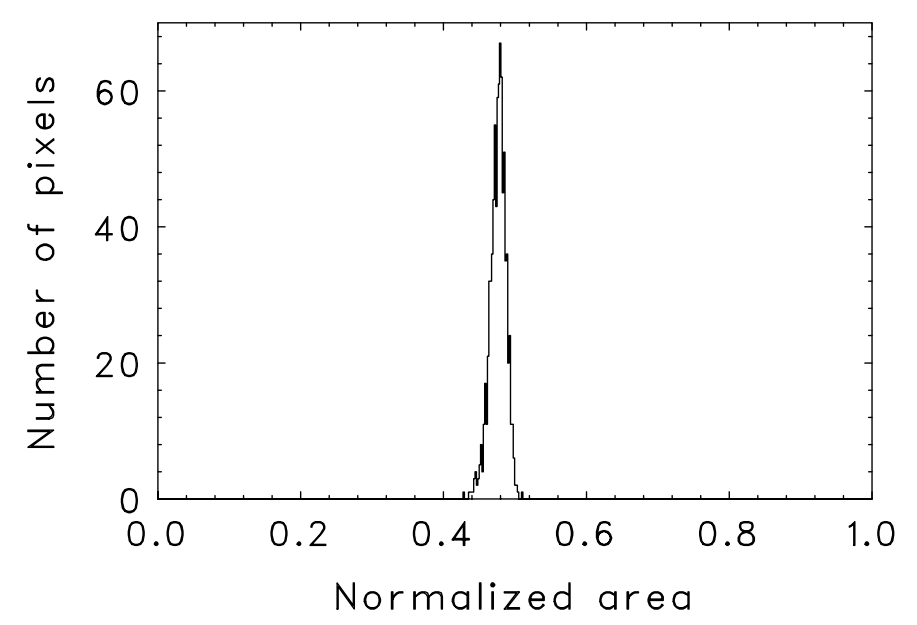

Figure 16. Distribution for the $60 \mathrm{keV}$ peak area normalized by the total counts. The variation of the distribution is $2.1 \%$.

$\mathrm{keV}$. Although the counts are different, the shape of the spectra is very similar. This similarity implies that the variation of the peak area or the total count rate is not caused by the spatial inhomogeneity of charge transport properties, such as mobility and lifetime. This variation then could be explained by two possibilities. One is a reduction of the effective area caused by the bump bonding process. The other is the dead time variations due to the algorithm currently installed in the data acquisition (DAQ) system. We will study this issue more.

If we assume that the variation of total counts is due to the dead-time variations for each pixel, we can use it as an indicator of the actual integration time for each pixel. Figure 15 shows a map of the "normalized" area (area ratio). In the figure, the integrated counts from $45 \mathrm{keV}$ to $65 \mathrm{keV}$ are normalized by the total counts of the spectrum for each pixel. The resultant variation of the area ratio is $2.1 \%$ while the statistical error is $1.3 \%$.

\section{IMAGING SPECTROSCOPY}

We perform imaging measurements to demonstrate the performance of imaging spectroscopy. An image mask, made of brass covered with gold, is mounted $6 \mathrm{~mm}$ above the detector. It has a length of $70 \mathrm{~mm}$ and a thickness 
of $0.8 \mathrm{~mm}$. The area marked in Fig. 17 (a) is used for the measurement. ${ }^{241} \mathrm{Am}$ and ${ }^{57} \mathrm{Co}$ are located $40 \mathrm{~cm}$ above the detector so that the shadow image is obtained with the CdTe pixel detector. The detector is operated at $-20{ }^{\circ} \mathrm{C}$ and $80 \mathrm{~V}$ for the pixel is applied.

As shown in Fig. 17 (b) and (c), we have succeeded in imaging spectroscopy using the detector. Fig. 17 (b) is drawn by $13 \mathrm{keV}-26 \mathrm{keV}$ lines from ${ }^{241} \mathrm{Am}$, while Fig. 17 (c) is by $122 \mathrm{keV}$ line from ${ }^{57}$ Co. The spoke feature of the mask, which is the same as the pixel size of $500 \mu \mathrm{m}$, is clearly resolved while a featureless image is obtained for the image selected from the $105-130 \mathrm{keV}$ energy band. This result is consistent with the fact that the mask is transparent above $100 \mathrm{keV}$.

\section{(a) mask photo}

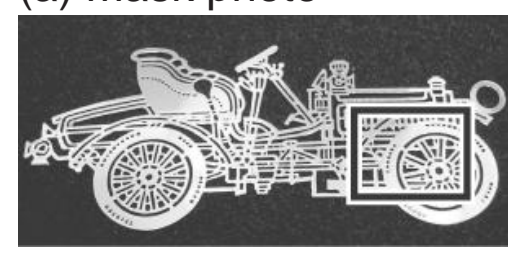

(b) $13-26 \mathrm{keV}$

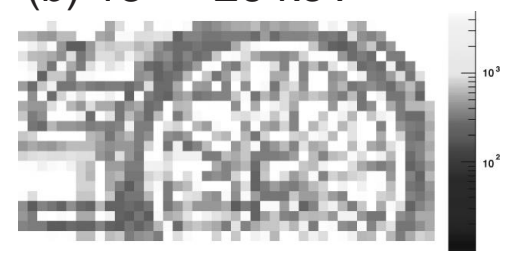

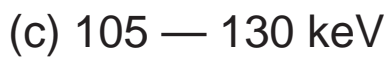

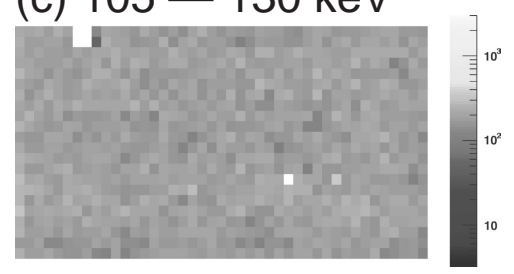

Figure 17. (a) Photo of a mask, and images using (b) $13 \mathrm{keV}-26 \mathrm{keV}$ lines of ${ }^{241} \mathrm{Am}$ and (c) the $122 \mathrm{keV}$ line of ${ }^{57} \mathrm{Co}$, respectively. The mask is made of brass covered with gold. A rectangular area is used in the measurement.

\section{SUMMARY}

We have developed a large CdTe pixel detector by utilizing recent technologies of an uniform CdTe single crystal, the two-dimensional ASIC, and the In/Au stud bump bonding. When the detector is operated at a temperature of $-50{ }^{\circ} \mathrm{C}$, the energy resolution (FWHM) for the $14 \mathrm{keV}$ and $60 \mathrm{keV} \gamma$-rays is $0.67 \mathrm{keV}$ and 0.89 $\mathrm{keV}$, respectively. The voltage scanning measurement shows that $10 \%$ of the total event become multi-pixel events, which contributes to the tail structure in thespectra obtained. The detector shows high uniformity as well as the spectral and imaging performances. The location of the peak agrees to within $0.82 \%$ after the analog gain is calibrated by pulser data, and the average energy resolution is $1.04 \mathrm{keV}$. The variation of the $60 \mathrm{keV}$ peak counts is found to be $8.9 \%$, which is inconsistent with the result for the planar CdTe detector. Although the reason for this variation is still under investigation, if we assume a "normalized" area, the resultant variation is $2.1 \%$. In the demonstration of imaging spectroscopy, the detector resolves a $0.5 \mathrm{~mm}$ wide line, which is the same as the positional resolution.

\section{ACKNOWLEDGMENTS}

The authors would like to thank M. Onishi for his dedicated help throughout the detector development. 


\section{REFERENCES}

1. K. Yamashita, et al., "Supermirror hard-x-ray telescope," Applied Optics, vol. 37, pp. 8067-8073, 1998

2. T. Takahashi, K. Makishima, F. Fukazawa, M. Kokubun, K. Nakazawa, M. Nomachi, H. Tajima, M. Tashiro, and Y. Terada, "Hard X-ray and Gamma-ray detectors for the next mission,", New Astronomy Reviews, vol 48, pp. 309-313, 2004

3. H. Kunieda, "Hard X-ray Telescope Mission (NeXT)," Proc. SPIE, vol. 5488, in press, 2004

4. F. A. Harrison, et al., "Technology development for the Constellation-X hard-x-ray telescope," in Proc. SPIE, vol. 3765, pp. 104-111, 1999

5. W. Craig, et al., "NuSTAR," in Proc. SPIE, vol 5488, in press, 2004

6. NeXT Satellite Proposal,the NeXT working group, submitted to ISAS, 2003.

7. Y. Ogasaka, K. Tamura, R. Shibata, A. Furuzawa, T. Okajima, K. Yamashita, Y. Tawara, H. Kunieda, "NeXT Hard X-ray Telescope," Proc. SPIE, vol. 5488, in press, 2004

8. T. Takahashi, et al., "Wide-band X-ray Imager (WXI) and Soft Gamma-ray Detector (SGD) for the NeXT mission," Proc. SPIE, vol. 5488, 2004

9. F. A. Harrison, S. E. Boggs, A. E. Bolotnikov, C. M. H. Chen, W. R. Cook and S. M. Schindler, "Development of CdZnTe pixel detectors for astrophysical applications", Proc. SPIE, vol. 4141, pp. 137-143, 2000

10. G. Sato, T. Takahashi, M. Sugiho, M. Kouda, T. Mitani, K. Nakazawa, Y. Okada, and S. Watanabe, "Characterization of CdTe/CdZnTe detectors," IEEE Trans. Nucl. Sci., vol 49, No. 3, pp. 1258-1263, 2002

11. M. Suzuki, et al. "Hard X-ray Response of CdZnTe Detectors in the Swift Burst Alert Telescope," IEEE Trans. Nucl. Sci., in print, 2003

12. M. Funaki, et al., "Growth and Characterization of CdTe Single Crystal for Radiation Detectors," Nucl. Instrum. Methods., vol. A436, pp. 120-126, 1999

13. T. Takahashi, T. Mitani, Y. Kobayashi, M. Kouda, G. Sato, S. Watanabe, K. Nakazawa, Y. Okada, M. Funaki, R. Ohno, and K. Mori, "High Resolution Schottky CdTe Diodes," IEEE Trans. Nucl. Sci., 49, No. 3 , pp. 1297-1303, 2002

14. K. Nakazawa, T. Takahashi, S. Watanabe, G. Sato, M. Kouda, Y. Okada, T. Mitani, Y. Kobayashi, Y. Kuroda, M. onishi, R. Ohno, and A. Kitajima, "Large-area CdTe diode detector for space application," Nucl. Instrum. Methods, vol. A512, pp. 412-418, 2003

15. T. Mitani, T. Tanaka, K. Nakazawa, T. Takahashi, T. Takashima, H. Tajima, H. Nakamura, M. Nomachi, T. Nakamoto, Y. Fukazawa "A Prototype Si/CdTe Compton Camera and the Polarization Measurement", IEEE Trans. Nucl. Sci., in press, 2004

16. T. Tanaka et al., "Development of a Si/CdTe semiconductor Compton Telescope," Proc. SPIE, vol. 5501, in press, 2004

17. C. M. H. Chen, et al. "Characterization of the HEFT CdZnTe pixel detectors," Proc. SPIE, vol. 5198, pp. 9-18, 2003

18. T. Takahashi, et al., "High-Resolution CdTe Detector and Applications to Imaging Devices", IEEE Trans. Nucl. Sci., 48, pp. 287-291, 2001

19. T. Takahashi and S. Watanabe "Recent Progress on CdTe and CdZnTe detectors," IEEE Trans. Nucl. Sci., vol. 48, pp. 950-959, 2001

20. F. Harrison, et al., "Development of a high spectral resolution cadmium zinc telluride pixel detector for astrophysical applications," Proc. SPIE, vol 4851, pp. 823-830, 2003 